\title{
Supplementary Information to
}

\section{“The Doping Bottleneck in Hematite: Multipole Clustering by Small Polarons"}

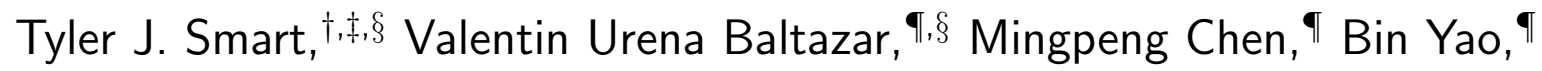 \\ Kiley Mayford, ${ }^{\dagger}$ Frank Bridges, ${ }^{\dagger}$ Yat Li, ${ }^{\mathbb{\top}}$ and Yuan Ping*, \\ $\dagger$ Department of Physics, University of California, Santa Cruz, CA, 95064, USA \\ $\ddagger$ Lawrence Livermore National Laboratory, Livermore, California 94551, USA \\ IDepartment of Chemistry and Biochemistry, University of California, Santa Cruz, CA, \\ 95064, USA \\ $\S$ Contributed equally to this work \\ E-mail: yuanping@ucsc.edu
}

\section{Supercell Convergence}

Various quantities were computed in a larger supercell $(3 \times 3 \times 1,270$ atoms $)$, to estimate the uncertainty in our $2 \times 2 \times 1$ results due to the finite supercell size. The ionization energy, formation energy, and quadrupole binding energy are all tabulated in SI Table S1. We note the results changed by 10 to $50 \mathrm{meV}$ between $2 \times 2 \times 1$ and $3 \times 3 \times 1$. All the major trends discussed in the manuscript still hold here. For example, Sn owns the smallest binding energy and Ti owns the largest binding energy. 
Table S1. Comparison of the calculated ionization energies $I E$, formation energies $E^{f}$ (at $q=0$ ), and quadrupole binding energies $\Delta_{\text {quad }}$ computed with $2 \times 2 \times 1$ and $3 \times 3 \times 1$ supercells. All values are in $\mathrm{eV}$.

\begin{tabular}{|c|c|c|c|c|c|c|c|c|}
\hline & Supercell Size & Ge & $\mathrm{Hf}$ & $\mathrm{Pb}$ & $\mathrm{Si}$ & Sn & $\mathrm{Ti}$ & $\mathrm{Zr}$ \\
\hline \multirow[t]{2}{*}{$I E($ dipole $)$} & $2 \times 2 \times 1$ & 0.197 & 0.267 & 0.242 & 0.165 & 0.255 & 0.157 & 0.259 \\
\hline & $3 \times 3 \times 1$ & 0.189 & 0.317 & 0.266 & 0.143 & 0.258 & 0.163 & 0.305 \\
\hline \multirow[t]{2}{*}{$I E_{1}(q u a d)$} & $2 \times 2 \times 1$ & 0.287 & 0.195 & 0.181 & 0.376 & 0.164 & 0.308 & 0.205 \\
\hline & $3 \times 3 \times 1$ & 0.280 & 0.220 & 0.225 & 0.343 & 0.200 & 0.309 & 0.248 \\
\hline \multirow[t]{2}{*}{$I E_{2}($ quad $)$} & $2 \times 2 \times 1$ & 0.484 & 0.463 & 0.416 & 0.343 & 0.449 & 0.361 & 0.459 \\
\hline & $3 \times 3 \times 1$ & 0.401 & 0.490 & 0.476 & 0.363 & 0.486 & 0.353 & 0.466 \\
\hline \multirow[t]{2}{*}{$E^{f}($ dipole $)$} & $2 \times 2 \times 1$ & 1.255 & 1.587 & 1.506 & 2.393 & 1.327 & 1.329 & 1.675 \\
\hline & $3 \times 3 \times 1$ & 1.249 & 1.597 & 1.514 & 2.363 & 1.348 & 1.323 & 1.682 \\
\hline \multirow[t]{2}{*}{$E^{f}(q u a d)$} & $2 \times 2 \times 1$ & 2.394 & 3.120 & 2.972 & 4.560 & 2.626 & 2.425 & 3.270 \\
\hline & $3 \times 3 \times 1$ & 2.400 & 3.136 & 2.973 & 4.561 & 2.657 & 2.435 & 3.285 \\
\hline \multirow[t]{2}{*}{$\Delta_{\text {quad }}$} & $2 \times 2 \times 1$ & -0.117 & -0.055 & -0.039 & -0.227 & -0.029 & -0.233 & -0.080 \\
\hline & $3 \times 3 \times 1$ & -0.099 & -0.057 & -0.055 & -0.164 & -0.039 & -0.212 & -0.080 \\
\hline
\end{tabular}


a

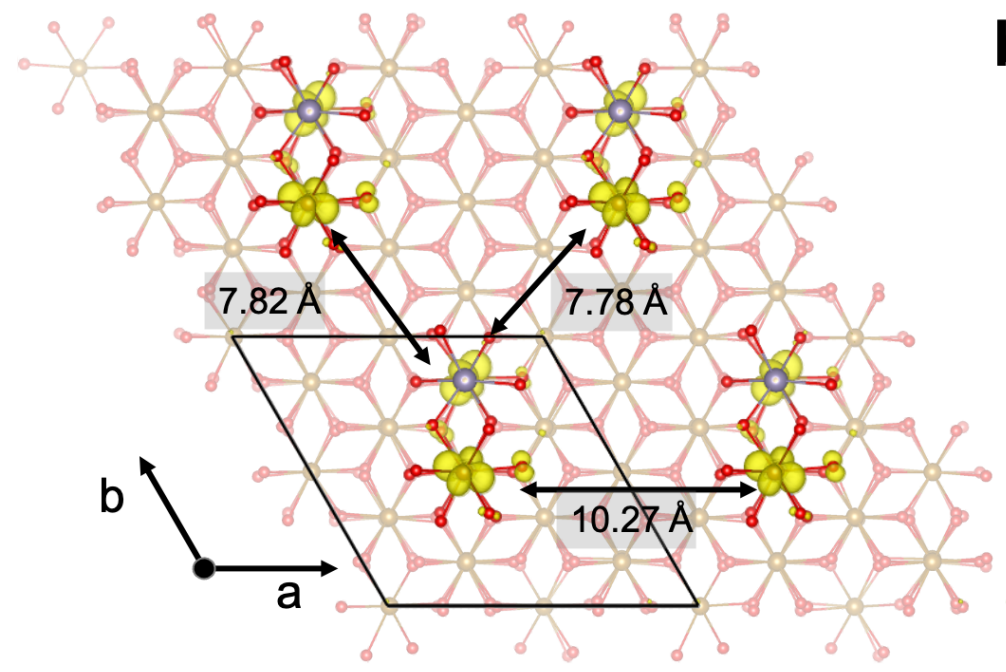

c

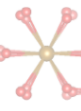
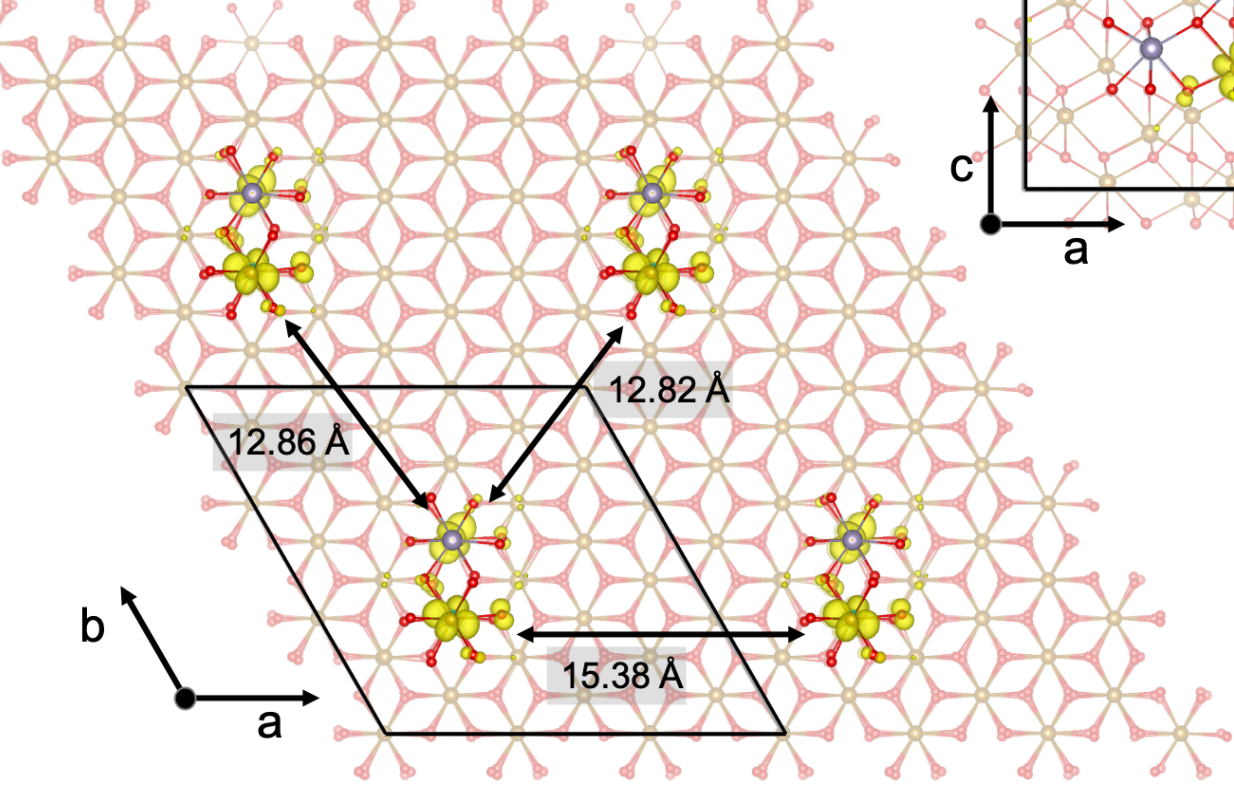

Figure S1. Distances between periodic images of the Sn quadrupole in the (a) ab plane of the $2 \times 2 \times 1$ supercell, (b) ac plane of the $2 \times 2 \times 1$ supercell, and (c) ab plane of the $3 \times 3 \times 1$ supercell. Distances represent the smallest distance between any polaron or Sn dopant of one quadrupole and another periodic image.

\section{Elemental Chemical Potentials}

The chemical potential of $\mathrm{Fe}$ and $\mathrm{O}$ was evaluated from first principles following the approach employed in Ref. 1. Namely, for $\mathrm{Fe}_{2} \mathrm{O}_{3}$ in thermodynamic equilibrium growth conditions, 
the chemical potentials $\mu_{\mathrm{Fe}}$ and $\mu_{\mathrm{O}}$ must satisfy Eq. $1-3$ :

$$
\begin{array}{r}
2 \Delta \mu_{\mathrm{Fe}}+3 \Delta \mu_{\mathrm{O}}=\Delta H_{\mathrm{Fe}_{2} \mathrm{O}_{3}} \\
2 \Delta \mu_{O} \leq \Delta H_{\mathrm{O}_{2}} \\
3 \Delta \mu_{\mathrm{Fe}}+4 \Delta \mu_{\mathrm{O}} \leq \Delta H_{\mathrm{Fe}_{3} \mathrm{O}_{4}}
\end{array}
$$

where $\Delta \mu_{\mathrm{i}}$ is the chemical potential of species $i$ referenced to its most stable phase. In an $\mathrm{O}$ rich environment, $\mathrm{Fe}_{2} \mathrm{O}_{3}$ will be in equilibrium with $\mathrm{O}_{2}$ as in Eq. 2, whereas in an $\mathrm{O}$ poor environment (Fe rich), $\mathrm{Fe}_{2} \mathrm{O}_{3}$ will be in equilibrium with $\mathrm{Fe}_{3} \mathrm{O}_{4}$ as in Eq. 3. In order to correct the well-known overbinding problem of $\mathrm{O}_{2}$ by DFT, we use the experimental value of $5.23 \mathrm{eV}$ for the binding energy of $\mathrm{O}_{2}$ in order to correct the total energy of $\mathrm{O}_{2}$ computed by DFT. ${ }^{2}$ Meanwhile, the chemical potential of each tetravalent dopant $X$ was computed as in Ref. 3, where after obtaining the chemical potential of $\mathrm{O}$ in the rich/poor limit, the chemical potential $\Delta \mu_{\mathrm{X}}$ can be computed from Eq. 4.

$$
\Delta \mu_{X}+2 \Delta \mu_{\mathrm{O}} \leq \Delta H_{X \mathrm{O}_{2}}
$$

\section{Defect Concentration}

In order to relate the effects of doping directly to polaron concentrations, we can compute the defect concentrations self-consistently by evaluating charge neutrality based on the formation energy of each defect. Following the formalism presented in Ref. 4, the charged defect concentration $\left(c_{q}\right)$ is computed as:

$$
c_{q}\left(X ; \varepsilon_{F}\right)=g \exp \left[-E_{q}^{f}\left(X ; \varepsilon_{F}\right) / k_{B} T\right]
$$

where $g$ is the degeneracy factor accounting for the internal degrees of freedom of the point defect, $k_{B}$ is the Boltzmann factor, and $T$ is temperature. In order to maintain neutrality, 
the introduction of defect $X$ with charge $q$ into the lattice must be compensated by defects of opposing charge or through the generation of free carriers. Specifically charge neutrality must be held:

$$
\sum_{X, q} c_{q}\left(X ; \varepsilon_{F}\right)+n_{h}-n_{e}=0
$$

where the concentration of free delocalized holes $\left(n_{h}\right)$ and free delocalized electrons $\left(n_{e}\right)$ can be evaluated via:

$$
n_{e}-n_{h}=\int_{-\infty}^{\infty} d E \frac{D(E)}{1+\exp \left[\left(E-\varepsilon_{F}\right) / k_{B} T\right]}
$$

Here $D(E)$ is the electronic density of states of the pristine system. Eq. 6 can be evaluated by standard root-finding algorithms to obtain $\varepsilon_{F}$ where charge neutrality is held. Note, in hematite free electrons will localize into small polarons, and so the formation of free electron small polarons is entered in a similar way to a defect, i.e. with a formation energy. Finally, in order to relate to experimental measurements of $\mathrm{Fe}_{2} \mathrm{O}_{3}$ photoanodes, we computed the concentration first at a synthesis temperature of $T_{S}=1073 \mathrm{~K}\left(800{ }^{\circ} \mathrm{C}\right.$ is a common synthesis temperature ${ }^{5,6}$ ), and then recomputed charge neutrality at normal operation (room) temperature $T_{O}=300 \mathrm{~K}$ while maintaining defects total concentrations similar to methods employed by Ref. 1 .

\section{Most Stable Two Dopant Configuration}

In order to determine the most stable two-dopant configurations where dopants are placed close together, we computed the total energies of various Sn-Sn configurations where the dopants are within about $6 \AA$ of each other. Considered positions of the second $\mathrm{Sn}$ is shown in SI Figure S2. The results of these calculations are tabulated in SI Table S2. The lowest energy configuration, corresponding to the $4^{\text {th }}$ nearest neighbor, is the one studied in the 
main text.

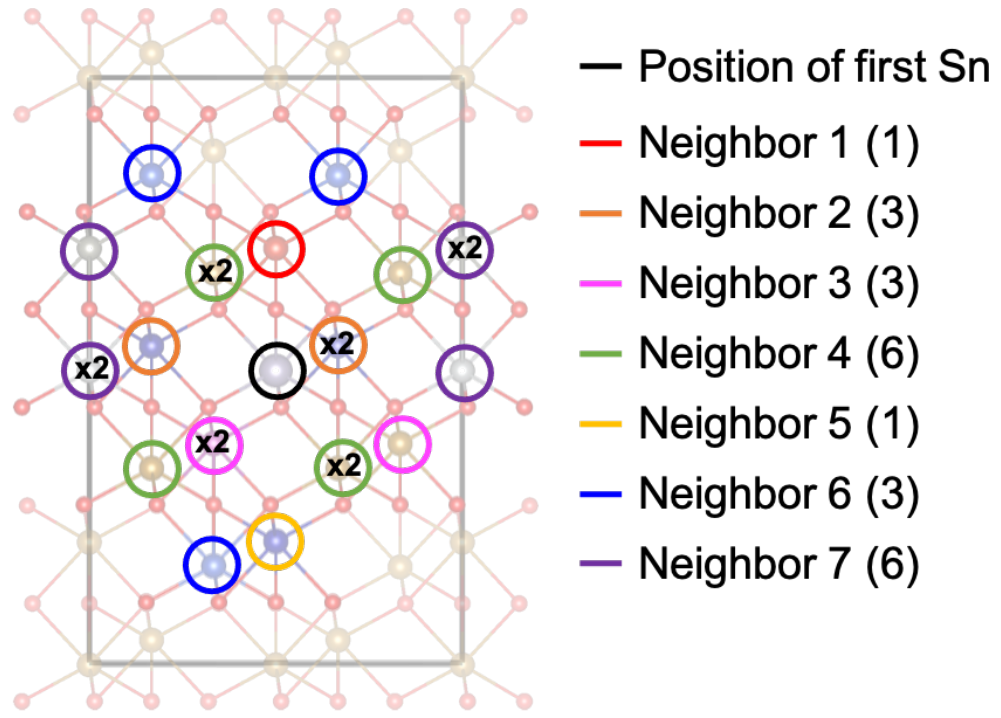

Figure S2. Positions of two Sn atoms correspond to those listed in SI Table S2. ' $x 2$ ' indicates there is a second neighbor not visible in the image which is directly behind the displayed one. On the right, ' $(g)$ ' denotes the degeneracy $g$ of each neighbor, which is also tabulated in SI Table S2, second column.

Table S2. Degeneracy, Sn-Sn distance, and relative total energy of various two Sn-dopant calculations (total energies $\Delta E$ are referenced to the nearest Sn-Sn configuration at $2.92 \AA$ ). Placing the second Sn at neighbor 4 gives the lowest energy, which is considered as the most stable configuration and used in the main text.

\begin{tabular}{c|ccc}
\hline \hline Neighbor & Degeneracy & Distance $(\AA)$ & $\Delta E(\mathrm{eV})$ \\
\hline 1 & 1 & 2.92 & 0.000 \\
2 & 3 & 3.02 & -0.412 \\
3 & 3 & 3.44 & -0.917 \\
4 & 6 & 3.77 & -1.111 \\
5 & 1 & 4.07 & -1.000 \\
6 & 3 & 5.13 & -0.967 \\
7 & 6 & 5.53 & -1.076 \\
\hline \hline
\end{tabular}




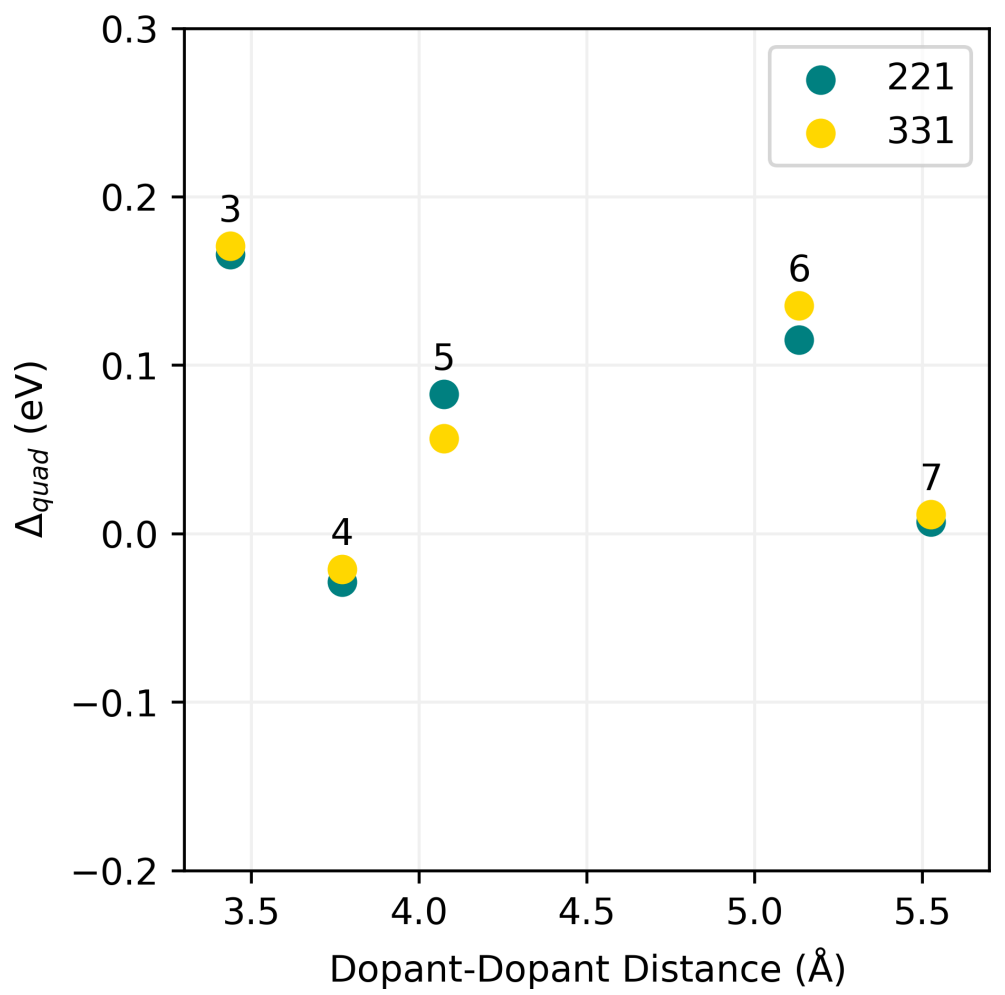

Figure S3. Binding energy $\left(\Delta_{\text {quad }}\right)$ of the $3^{\text {rd }}$ through $7^{\text {th }}$ neighbor for Sn-Sn pairs computed with a $2 \times 2 \times 1$ (teal) and $3 \times 3 \times 1$ (gold) supercells.

Table S3. Analogous to Table S2, but for the case of Ti. In this case Ti has a lowest energy configuration at a Ti-Ti distance of $5.13 \AA$.

\begin{tabular}{c|ccc}
\hline \hline Neighbor & Degeneracy & Distance $(\AA)$ & $\Delta E(\mathrm{eV})$ \\
\hline 1 & 1 & 2.92 & 0.000 \\
2 & 3 & 3.02 & -0.951 \\
3 & 3 & 3.44 & -0.954 \\
4 & 6 & 3.77 & -0.948 \\
5 & 1 & 4.07 & -0.137 \\
6 & 3 & 5.13 & -1.035 \\
7 & 6 & 5.53 & -0.972 \\
\hline \hline
\end{tabular}




\section{Electronic Structure of Tetravalent Dopants}

\section{Single Dopants (Dipole)}
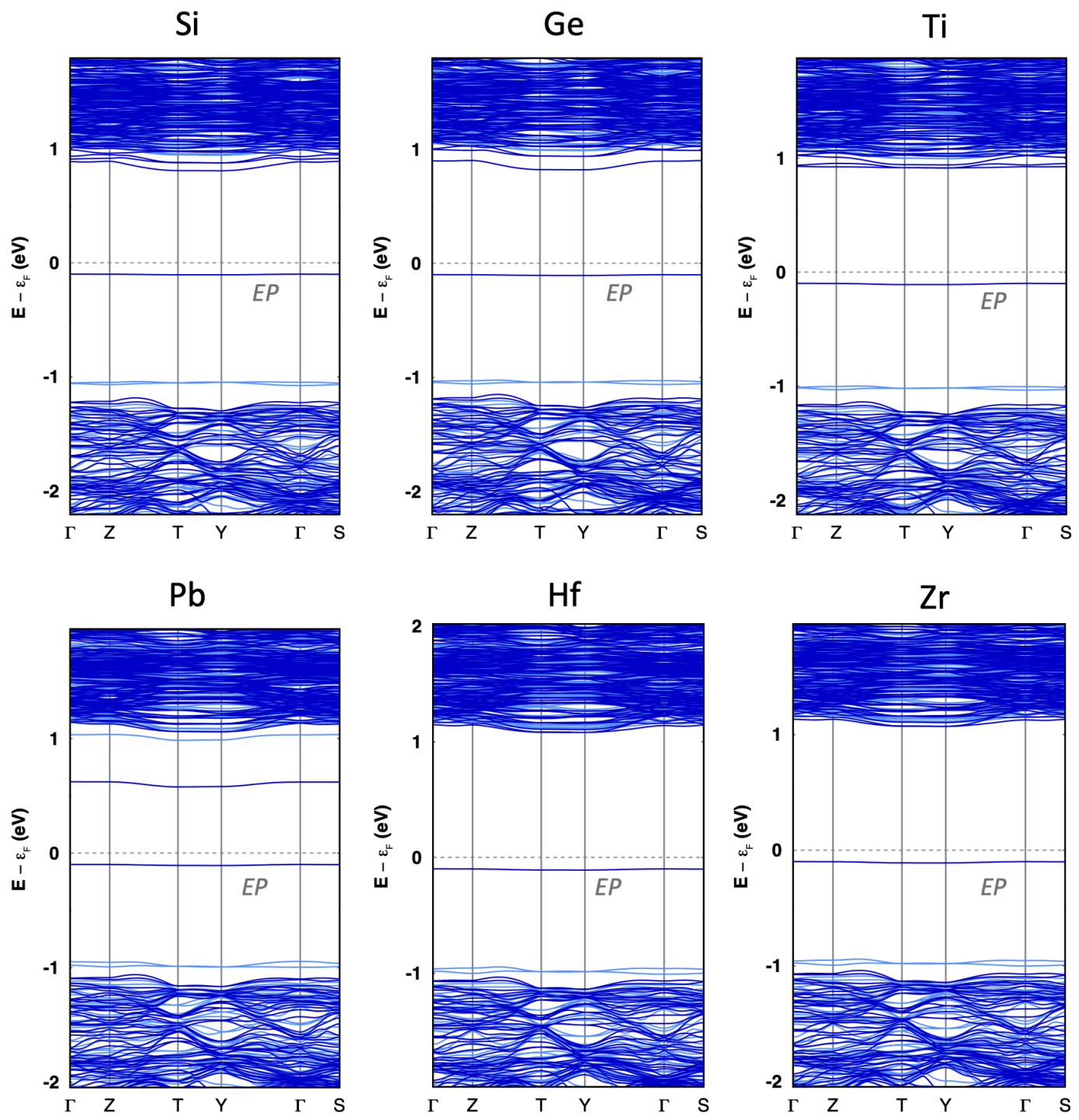

Figure S4. Band structure for $\mathrm{Fe}_{2} \mathrm{O}_{3}$ with single dopants studied in this work. The single polaron state is labeled by $E P$. 

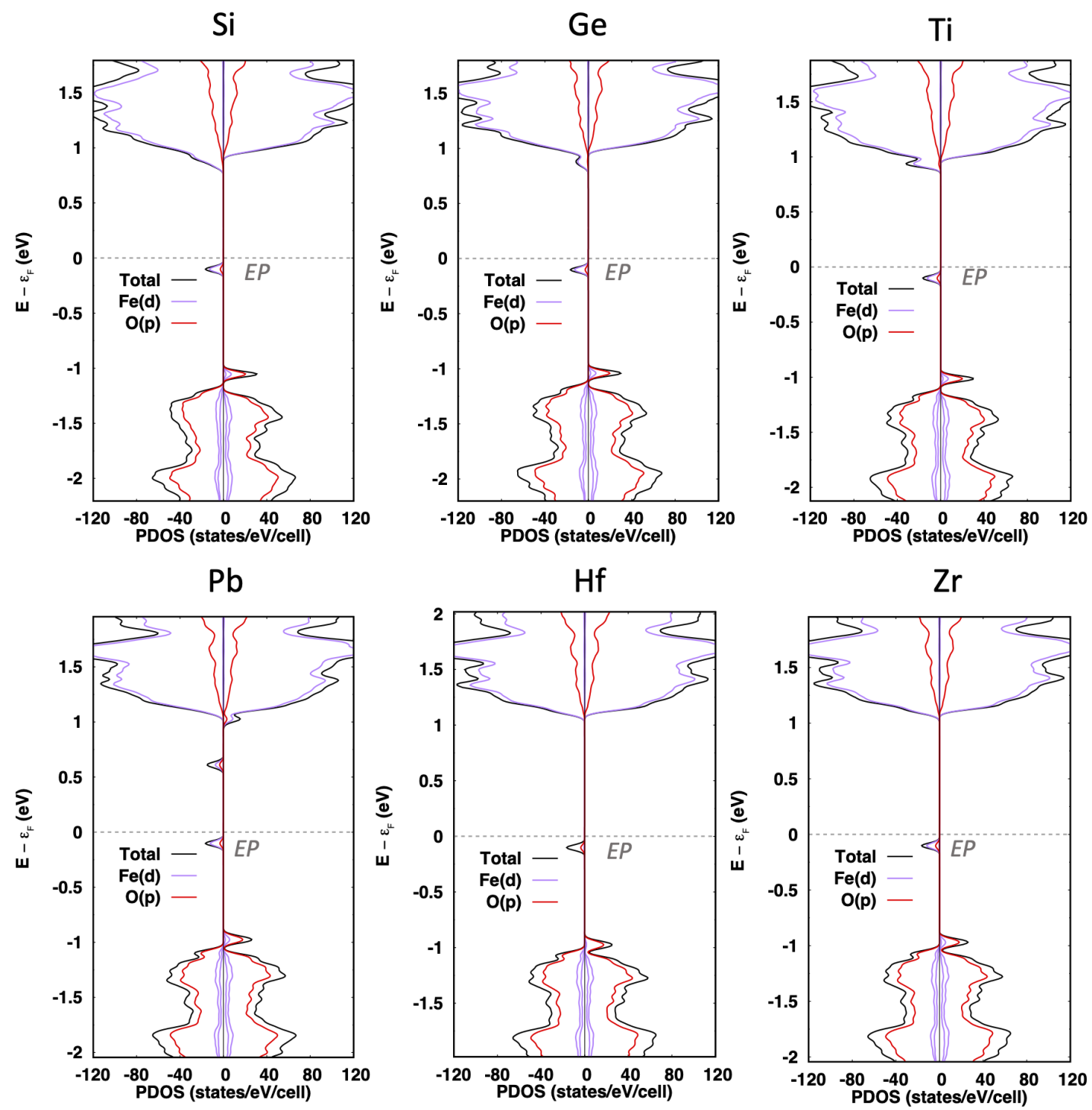

Figure S5. Projected density of states (PDOS) for $\mathrm{Fe}_{2} \mathrm{O}_{3}$ with single dopants studied in this work. The single polaron state is labeled by EP. 

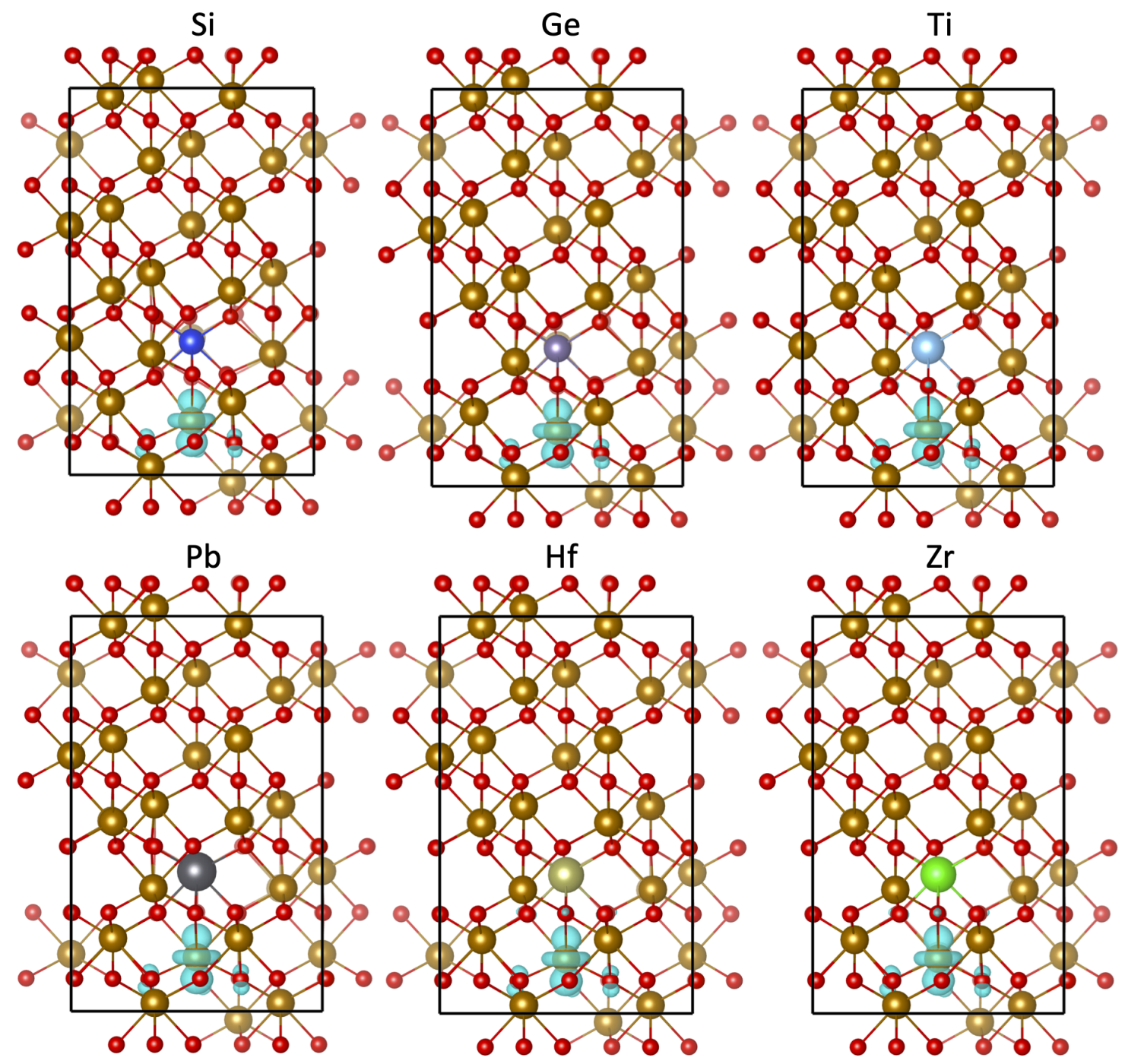

Figure S6. Crystal systems with single dopants in $\mathrm{Fe}_{2} \mathrm{O}_{3}$ studied in this work, where gold $=\mathrm{Fe}$, red $=\mathrm{O}$, and the remaining colored atom is the dopant labeled above each figure. The cyan cloud is the isosurface of the polaron wavefunction (the isosurface level is $1 \%$ of the maximum). 


\section{Two Dopants (Quadrupole)}
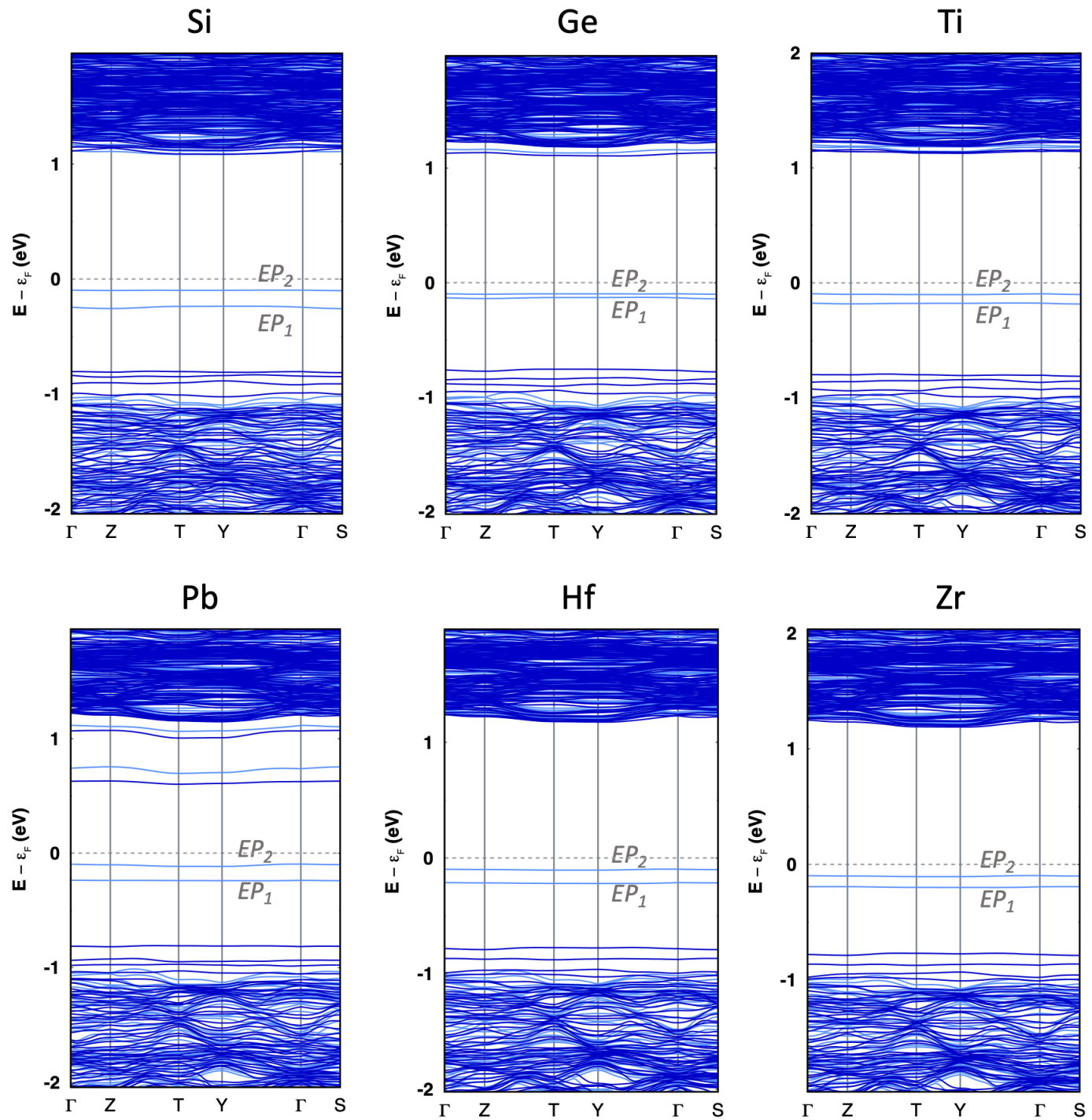

Figure S7. Band structure for $\mathrm{Fe}_{2} \mathrm{O}_{3}$ with quadrupole dopants studied in this work. The two small polaron states are labeled by $E P_{1}$ and $E P_{2}$. 

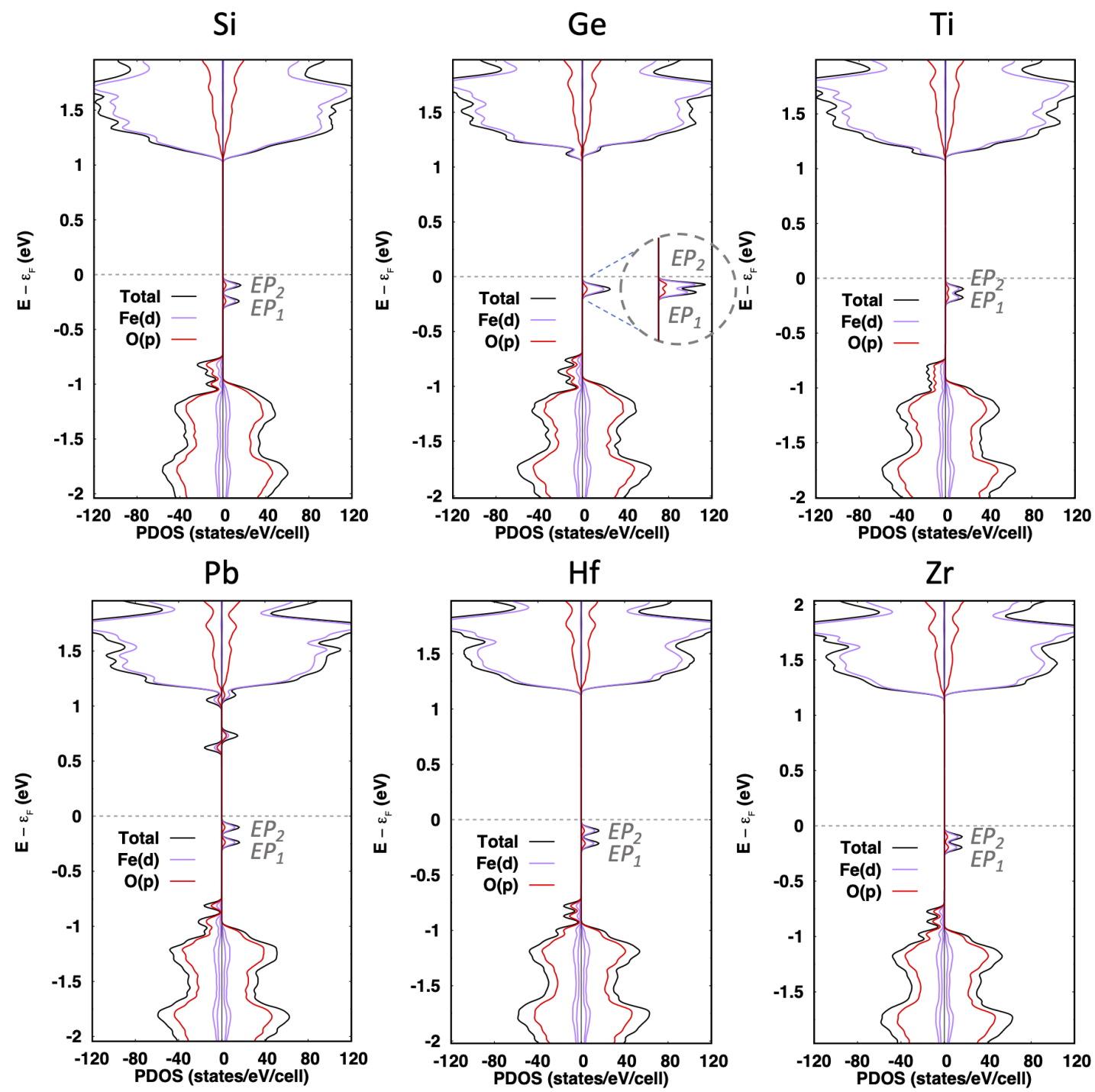

Figure S8. PDOS for $\mathrm{Fe}_{2} \mathrm{O}_{3}$ with quadrupole dopants studied in this work. The two small polaron states are labeled by $E P_{1}$ and $E P_{2}$. For Ge, an inset image is provided with less smearing and higher energy resolution to show that the states have a small energy splitting, e.g. $\sim 0.04 \mathrm{eV}$ at $\Gamma$. 

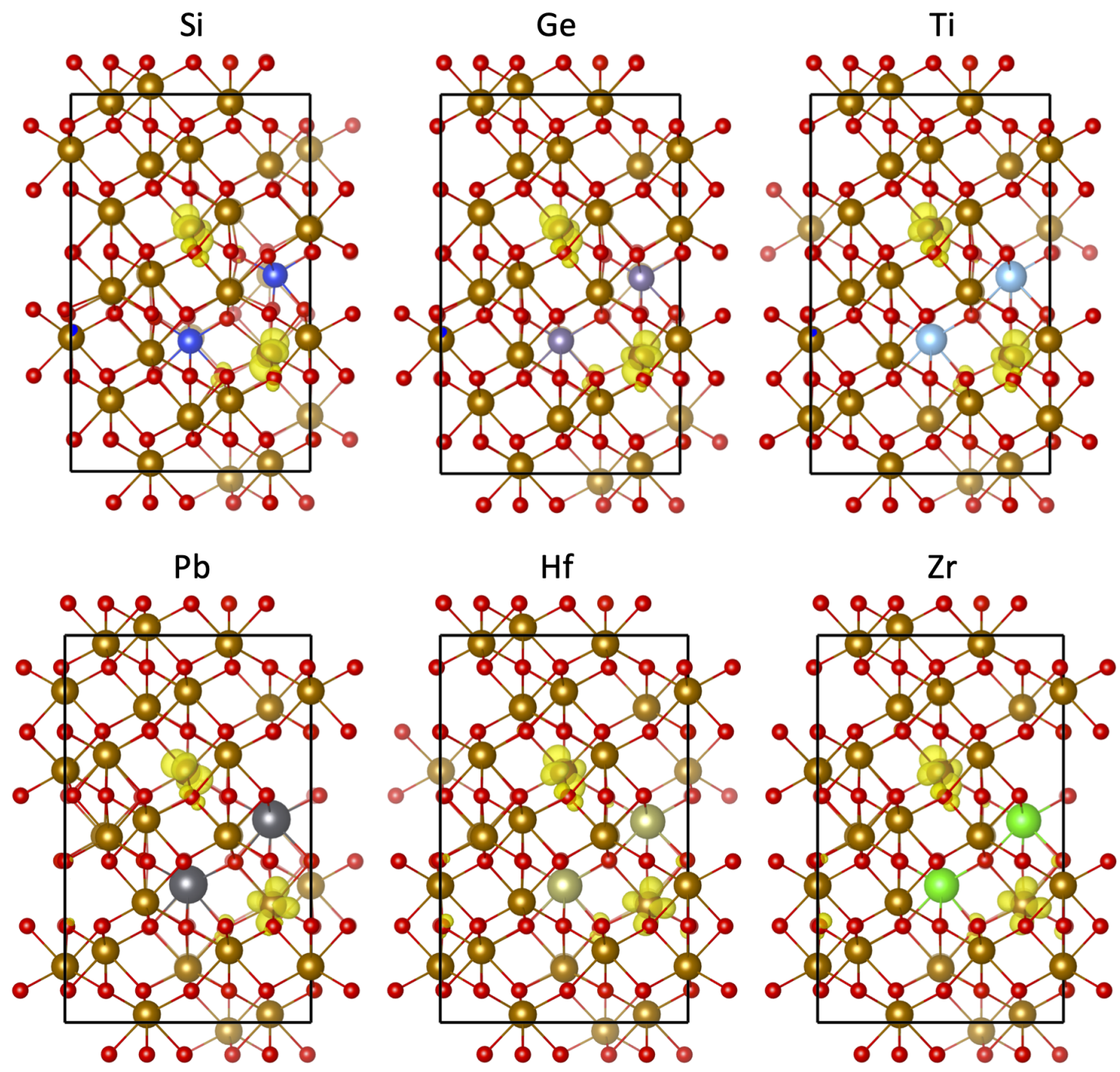

Figure S9. Crystal system for $\mathrm{Fe}_{2} \mathrm{O}_{3}$ with quadrupole dopants studied in this work. In each plot, their are two yellow clouds, corresponding to the two small electron polaron wavefunction (the isosurface level is $1 \%$ of the maximum). 


\section{Perturbed Conduction State from $\mathrm{Pb}$ Doping}
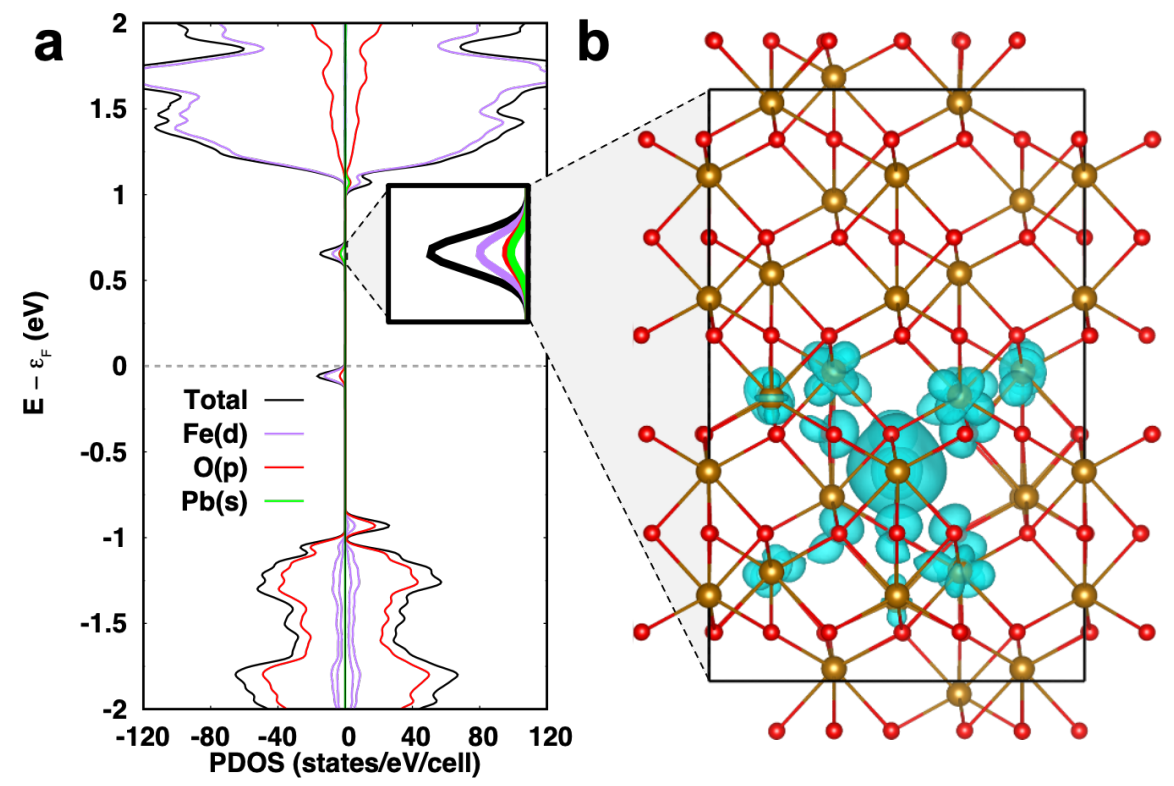

Figure S10. In the case of $\mathrm{Pb}$ doping, a state forms just below the conduction band which is primarily perturbed $\mathrm{Fe} 3 d$ orbitals but also a mixture of $\mathrm{Pb} 6 s$ and $\mathrm{O} 2 p$. This type of perturbative state was not seen in other doping cases besides $\mathrm{Pb}$.

\section{Synthesis of Sn-Doped $\mathrm{Fe}_{2} \mathrm{O}_{3}$ Samples}

Sn doped hematite nanowires were prepared by a two-step process. First, FeOOH nanowires were synthesized using hydrothermal method reported previously. ${ }^{5}$ FeOOH nanowire powders were mixed with $\mathrm{SnCl}_{4}$ ethanol solution. We adjusted the concentration of $\mathrm{SnCl}_{4}$ solution to control the Sn doping concentration. The powders were dried at $80{ }^{\circ} \mathrm{C}$ and then annealed in air at $550{ }^{\circ} \mathrm{C}$ for 30 min to convert $\mathrm{FeOOH}$ to hematite. To facilitate the diffusion of Sn dopant into the hematite crystal structure, the sample was further annealed at 800 ${ }^{\circ} \mathrm{C}$ for another $10 \mathrm{~min}$. These prepared $\mathrm{Sn}$ doped hematite samples were used for EXAFS characterization. 


\section{EXAFS Characterization of Sn-Doped $\mathrm{Fe}_{2} \mathrm{O}_{3}$}

The EXAFS data were collected on beamline 4-1 at the Stanford Synchrotron Radiation Lightsource (SSRL), using 220 Si monochromator crystals. At the Sn K edge, the monochromator was detuned $30 \%$ to reduce harmonics; the slits were $0.2 \times 6.0 \mathrm{~mm}^{2}$ providing an instrumental resolution of $\sim 3 \mathrm{eV}$, well below the core-hole lifetime value. The $\mathrm{X}$-ray data were reduced using the program $\mathrm{RSXAP}^{7}{ }^{7}$ which uses standard techniques to remove the pre- and post-backgrounds, and to extract the EXAFS oscillations as a function of $k$. Next, a fast Fourier transform (FFT) was used to transform the data to $r$-space, as plotted in Figure 2 in the main text.

The data were fit to a sum of pair-functions, calculated using FEFF7 ${ }^{8}$ for Sn substituted on an $\mathrm{Fe}$ site in undistorted $\mathrm{Fe}_{2} \mathrm{O}_{3}$. The initial distances started at the values for hematite. There are five Sn-O and four Sn-Fe peaks out to $3.8 \AA$; two very weak peaks near $4.0-4.1 \AA$ did not contribute significantly. In the first $\mathrm{O}$ shell of hematite there are two Fe-O distances, 1.944 and $2.116 \AA$; however, because $\mathrm{Sn}(4+)$ has a larger ionic radius than high-spin $\mathrm{Fe}(3+)$, the shortest Sn-O distance will be closer to $2.0 \AA$. Thus the splitting is expected to decrease. Initial attempts to fit to a split Sn-O first neighbor peak lead to very small splittings of order $0.05 \AA$. Because only splittings greater than $0.12 \AA$ can be resolved for an FT range up to 13 $\AA^{-1}$, a single Sn-O peak was used for the first shell in final fits from 1.0-4.2 $\AA$. The second and third shell Sn-O peaks, although split by $0.2 \AA$ in $\mathrm{Fe}_{2} \mathrm{O}_{3}$, also collapse to a single peak with a distance of $3.49 \AA$ - the same as the average of these distances in hematite. The shortest Sn-Fe distance $(\sim 2.90 \AA)$ is close to the next Sn-Fe peak at $2.97 \AA$ and cannot be resolved; these two peaks were therefore constrained together. Finally, one or two multi-scattering (MS) peaks were included.

For the $0.1 \%$ sample which has the higher amplitude in the $3-4 \AA$ range, 8 peaks were used which included two MS peaks, but the smaller MS peak was constrained; 14 parameters ( $r$ 's, and $\sigma^{\prime}$ s) were varied and 7.35 degrees of freedom remain using Stern's criteria. ${ }^{9}$ For the $1 \%$ sample the smaller MS peak was removed and a Sn-Sn peak added to model the change 
in structure between 3 and $3.5 \AA$. Here 16 parameters were varied, leaving 5.35 degrees of freedom. ${ }^{9}$

The real space plots of the $1 \%$ and $0.1 \%$ Sn samples show strong agreement in terms of the phase and real part of the Fourier transform in the restricted range from 0-3 $\AA$. However, in the range of 3-3.8 $\AA$, the $1 \%$ sampled exhibited a drastic change in phase and a significant decrease in amplitude as compared with the $0.1 \%$ sample, indicative of interference. This interference is likely between an out of phase Sn-Fe peak and Sn-Sn peak both occurring at $3.7 \AA$ This is evidenced by the fact that a fit considering only Sn-Fe peaks resulted in little agreement with the data in that region (Figure S11a), while a fit including the Sn-Sn peak and subsequently reducing the number of Sn-Fe peaks from 6 to 5 resulted in significant agreement with the data collected at SSRL (Figure S11b). Figure S11c shows the theoretical calculations of the Sn-Sn peak and the Sn-Fe peak, both occurring at 3.7A. As one can see, they are almost completely out of phase with each other. As well, the amplitude of the Sn-Sn peak is larger than the Sn-Fe peak. This destructive interference decreases the total amplitude and shifts the data to slightly larger radius as can be seen by comparing the $0.1 \%$ data with the $1.0 \%$ data in Figure 2 of the main text, over that region. 


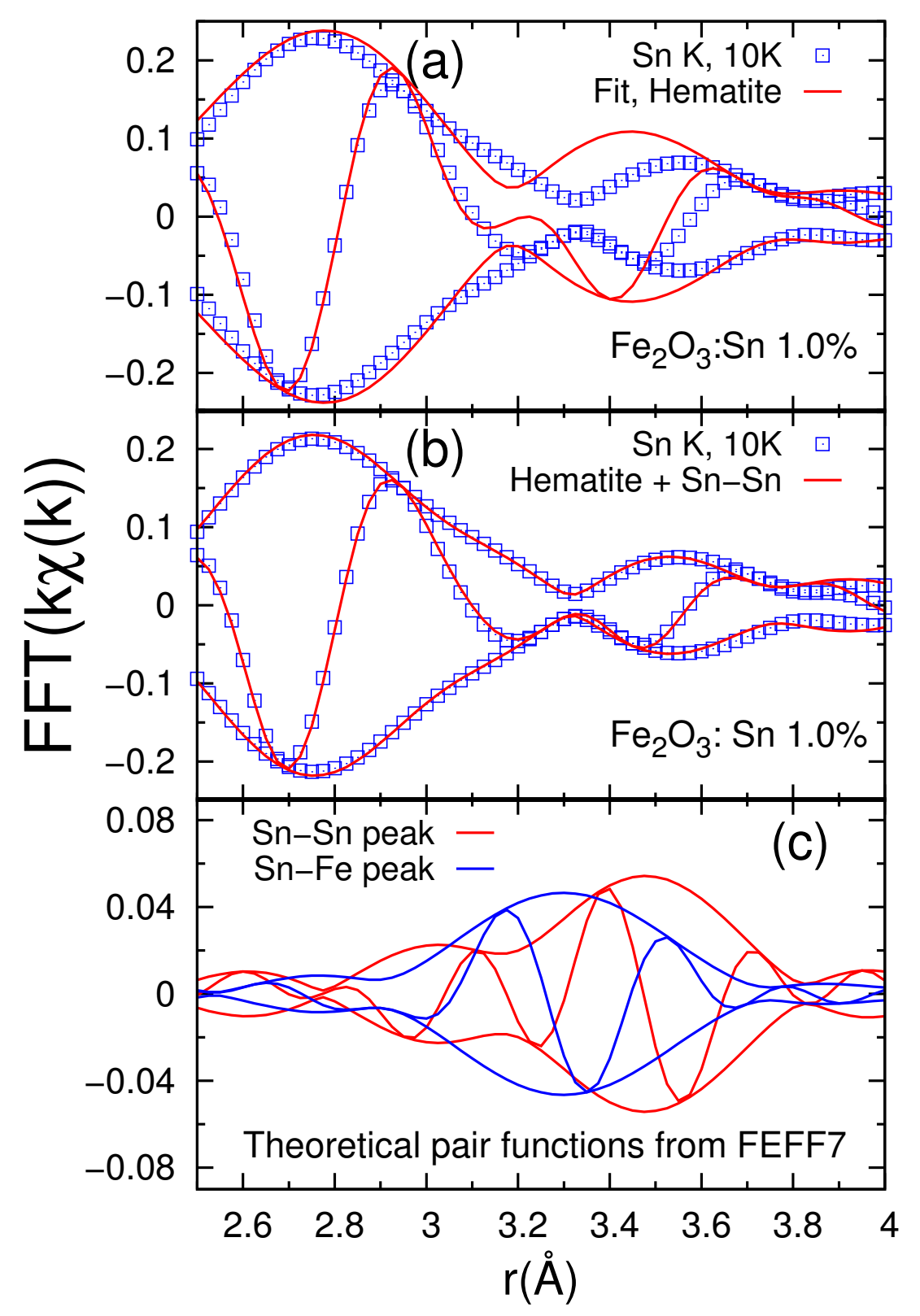

Figure S11. EXAFS r-space data at the K-edge for 1.0\% (a,b) and theoretical peaks calculated using FEFF7 (c) for the Sn-Sn and Sn-Fe pairs. Panel (a) was fit to the hematite structure and shows poor agreement with the data in the range 3.0-3.8 $\AA$. Panel (b) was fit to the hematite structure plus a Sn-Sn peak replacing a Sn-Fe peak and shows good agreement with the data. Panel (c) shows that the Sn-Fe and Sn-Sn real functions are nearly out of phase and hence destructively cancel.

The measured ratios of Sn/Fe for Sn doped hematite were obtained from the absorption step heights which are proportional to the number of atoms in the sample. Data were collected for each edge in transmission mode at the same point on the sample. The step 
heights of the Sn and Fe K edges were calculated using a linear fit above and below the edge. The absorption cross-sections per atom are tabulated in the McMaster tables as a function of energy. ${ }^{10}$ From these, the theoretical step heights per atom were calculated for each edge. The experimental step heights were normalized using these known step heights and the ratio of Sn to Fe in the sample was calculated using:

$$
\frac{S n}{F e}=\frac{\left(\frac{H_{e x p}}{H_{t h}}\right)_{S n}}{\left(\frac{H_{e x p}}{H_{t h}}\right)_{F e}}
$$

where $H_{e x p}$ is the experimental step height and $H_{t h}$ is the theoretical step height. The measured concentrations of Sn as compared with the nominal concentration are denoted in table S4.

Table S4. Measured concentrations of Sn in three samples as acquired by Eq. 8, compared with the nominal concentration.

\begin{tabular}{cc}
\hline \hline Nominal & Measured \\
\hline $1.0 \%$ & $0.76 \%$ \\
$0.3 \%$ & $0.215 \%$ \\
$0.1 \%$ & $0.091 \%$ \\
\hline \hline
\end{tabular}

\section{Probability of Quadrupole Formation}

Using a Maxwell-Boltzmann thermal distribution we compute the probability of forming a quadrupole, which we equate to the formation of a second dopant adjacent to another dopant based on their binding energy. At sufficiently high temperatures and low concentration, the Maxwell-Boltzmann distribution can describe the average distribution at thermal equilibrium. Following a statistical model we can compute the probability $P_{i}$ of forming state $i$ given by:

$$
P_{i}=\frac{g_{i} e^{-E_{i} / k T}}{Z} .
$$


Where $g_{i} e^{-E_{i} / k T}$ is the Boltzmann factor of state $i$ with energy $E_{i}$ and degeneracy $g_{i}$. Meanwhile, $Z$ is the partition function defined as:

$$
Z=\sum_{j=2}^{n} g_{j} e^{-E_{j} / k T}
$$

Here, $n$ is the number of unique Fe sites. The sum is taken from 2 to $n$ since in this approach we consider that one dopant has already formed, and we are predicting the probability distribution for the formation of the second dopant considering the dopant-dopant interaction (i.e. clustering). The total number of Fe sites $N$ is the sum of each site's degeneracy $\left(g_{j}\right)$ plus 1 (for the first site), i.e. $N=1+\sum_{j=2}^{n} g_{j}$. Therefore, the concentration is given by the number of dopants (2) over total number of sites: $c=2 / N$.

For quadrupole formation, we consider a simplified model wherein there is no interaction between the dopants $\left(E_{i}=0\right)$ except for when the dopant is placed at the quadrupole binding site. For this case, the energy is given by the binding energy of the dopant $\left(E_{\text {quad }}=\Delta_{\text {quad }}\right)$. Then the partition function defined above reduces to:

$$
Z=g_{q u a d} e^{-E_{q u a d} / k T}+\left(N-1-g_{q u a d}\right)
$$

where $g_{\text {quad }}$ is the degeneracy of the quadrupole configuration (6, as shown in SI Table S2). Using Eq. 9-11, we can compute the probability of forming a quadrupole as a function of dopant concentration at a given temperature $(T=500 \mathrm{~K}$ results are shown in SI Figure S13). In the end, this probability is used to combine the results of computing defect concentrations of single dopant and dopant-pair following the approach detailed in the above section. 


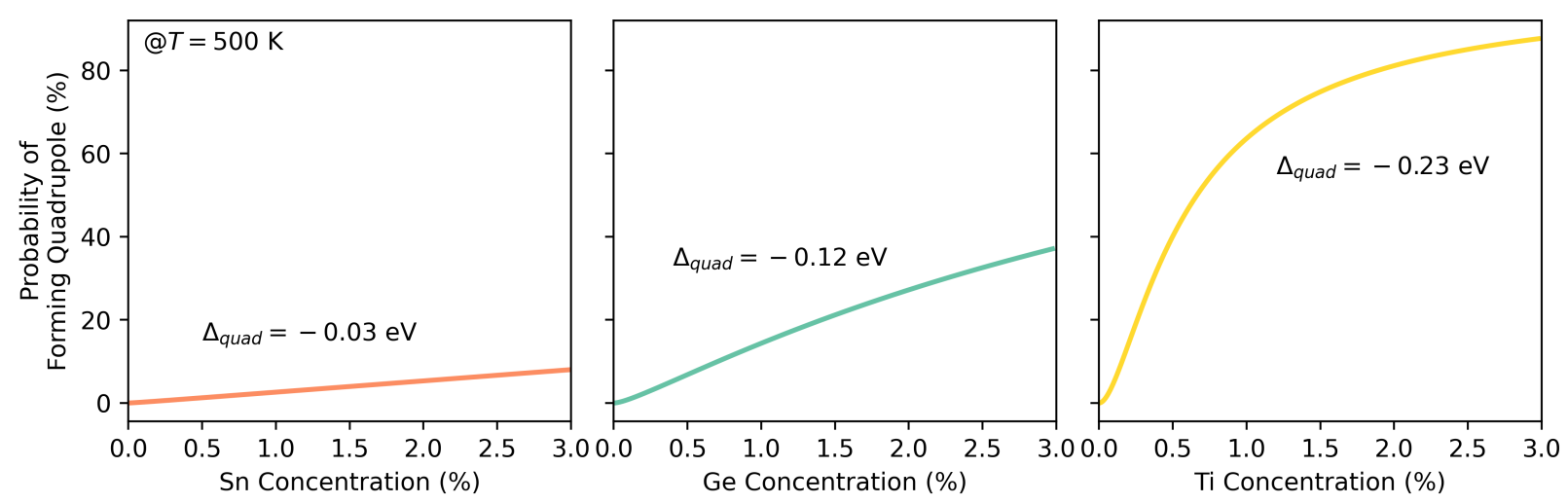

Figure S12. Computed probability of forming a quadrupole for various dopants computed by Eq. $9-11$ at $T=500 \mathrm{~K}$. 

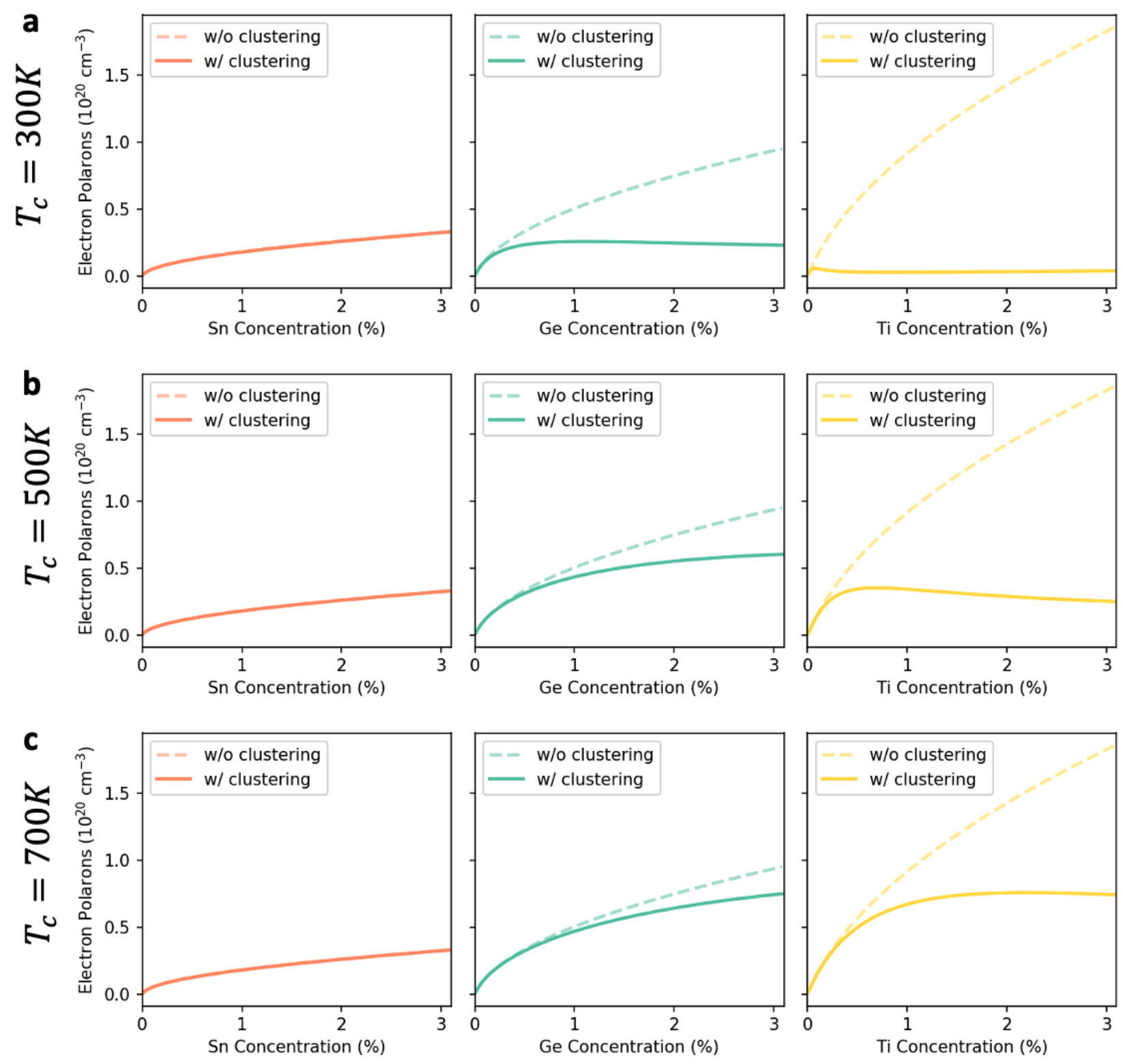

Figure S13. Computed free electron polaron concentration as a function of dopant concentration for $\mathrm{Sn}, \mathrm{Ge}$, and $\mathrm{Ti}$, with and without the effect of clustering (i.e. quadrupole formation) for various temperatures of clustering ( $T_{c}$, as in Eq. 9-11).

\section{Higher Order Multipoles}

In this work, we also examined higher order multipoles for $\mathrm{Sn}$ doped $\mathrm{Fe}_{2} \mathrm{O}_{3}$ as shown in SI Figure S14. We find that, similar to the quadrupole case, the arrangement of three Snpolaron pairs forms a 'hexapole', with a negative binding energy of $-59 \mathrm{meV}$. Here, we 
compute the binding energy of the hexapole by:

$$
\Delta_{h e x}=E^{f}(h e x)-E^{f}(q u a d)-E^{f}(\text { dipole }) .
$$

In this way, a negative binding energy indicates a favorable clustering of a quadrupole and a dipole into a hexapole.

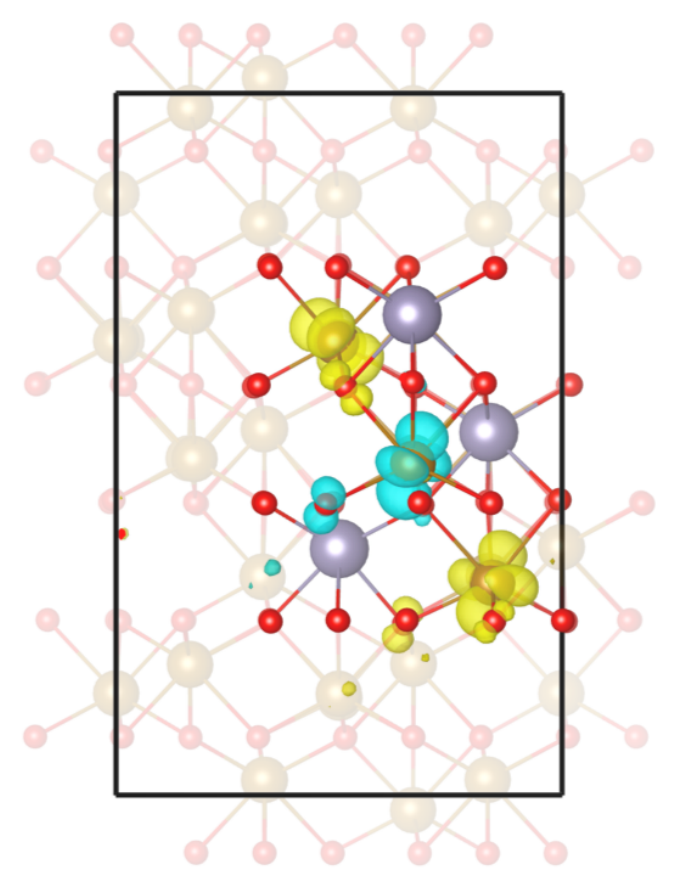

Figure S14. Three Sn dopants can cluster and form a higher order multipole along with three polaron states. The atoms are gold $=\mathrm{Fe}$, red $=\mathrm{O}$ and grey $=\mathrm{Sn}$. The yellow ( cyan (spin down) clouds are the isosurfaces of the polaron wavefunctions (the isosurface level is $1 \%$ of the maximum).

\section{References}

(1) Lee, J.; Han, S. Thermodynamics of native point defects in $\alpha-\mathrm{Fe}_{2} \mathrm{O}_{3}$ : an ab initio study. Physical Chemistry Chemical Physics 2013, 15, 18906-18914.

(2) Wang, L.; Maxisch, T.; Ceder, G. Oxidation energies of transition metal oxides within the GGA+ U framework. Physical Review B 2006, 73, 195107. 
(3) Zhou, Z.; Huo, P.; Guo, L.; Prezhdo, O. V. Understanding hematite doping with group IV elements: a DFT+ U study. The Journal of Physical Chemistry C 2015, 119, 26303-26310.

(4) Freysoldt, C.; Grabowski, B.; Hickel, T.; Neugebauer, J.; Kresse, G.; Janotti, A.; Van de Walle, C. G. First-principles calculations for point defects in solids. Reviews of Modern Physics 2014, 86, 253.

(5) Ling, Y.; Wang, G.; Wheeler, D. A.; Zhang, J. Z.; Li, Y. Sn-Doped Hematite Nanostructures for Photoelectrochemical Water Splitting. Nano Letters 2011, 11, 2119-2125.

(6) Tian, C.; Li, W.; Lin, Y.; Yang, Z.; Wang, L.; Du, Y.; Xiao, H.; Qiao, L.; Zhang, J.Y.; Chen, L., et al. Electronic Structure, Optical Properties and Photoelectrochemical Activity of Sn Doped $\mathrm{Fe}_{2} \mathrm{O}_{3}$ Thin Films. The Journal of Physical Chemistry C 2020, $124,12548-12558$.

(7) Booth, C. H. R-Space X-ray Absorption Package. 2010; http://lise.lbl.gov/RSXAP.

(8) Ankudinov, A. L.; Rehr, J. Relativistic calculations of spin-dependent x-ray-absorption spectra. Physical Review B 1997, 56, R1712.

(9) Stern, E. A. Number of relevant independent points in X-ray-absorption fine-structure spectra. Physical Review B 1993, 48, 9825.

(10) McMaster, W. H. Compilation of X-ray Cross Sections; UCRL-50174 Sec. II, Rev. 1; Lawrence Radiation Laboratory, University of California: Berkeley, 1969; url=http://books.google.com/books?id=8VHzmgEACAAJ. 